\title{
Planejamento pré-operatório de correção de deformidades supramaleolares através de impressão 3D
}

Tiago Baumfeld ${ }^{1}\left(\mathbb{D}\right.$, Ricardo Fernandes Rezende ${ }^{2} \mathbb{C}^{\text {, }}$, Luiza Helena Oliveira e Araújo ${ }^{1}$ (D), Marco Antonio Percope de Andrade $^{1}(\mathbb{D})$, Daniel Baumfeld ${ }^{1}(\mathbb{D})$, Rudolf Huebner ${ }^{1}$ (D)

\section{RESUMO}

Modelos impressos em 3D têm sido explorados profundamente no campo médico, destacando-se como importante ferramenta de auxílio para planejamento cirúrgico. Os autores apresentam relato de caso de um paciente, com artrose pós-traumática do tornozelo direito, submetido a osteotomia supramaleolar, em cunha de fechamento medial. Esta cirurgia foi realizada após planejamento operatório por impressão 3D, o que pode demonstrar reprodutibilidade deste método.

Palavras-chave: Tornozelo; Impressão tridimensional; Procedimentos cirúrgicos; Osteotomia. 


\section{INTRODUÇÃO}

A impressão tridimensional (3D) foi descrita pela primeira vez por Charles W. Hull em 1986, e tem sido extensivamente utilizada nos últimos 30 anos $^{1}$. Devido à sua reconstrução precisa de complexas estruturas anatômicas, é crescente o uso da impressão 3D em medicina, sendo utilizada para o aprendizado da anatomia básica à prática cirúrgica avançada². Modelos educativos incluindo ossos foram construídos utilizando tecnologia de impressão $3 D^{3}$. A maioria dos modelos impressos em 3D relatados são altamente precisos e realistas. Entretanto, apresentam limitações, principalmente relacionadas à escolha do hardware, software, e materiais a serem utilizados. Além disso, estes modelos podem não traduzir o dano da cartilagem articular, uma vez que são oriundos de um exame de Tomografia Computadorizada 2,4,5.

Atualmente, modelos impressos em 3D foram explorados profundamente no campo médico com vantagens de encurtar a curva de aprendizado para os cirurgiões e auxiliá-los no melhor planejamento de procedimentos cirúrgicos complexos ${ }^{2,3,5}$. Como exemplo, citamos o trabalho realizado por Feng Shuang et al., utilizando placas para osteossíntese personalizadas impressas em 3D, demonstrando a segurança e eficácia deste método para o tratamento de fraturas intercondilares do úmero, reduzindo significativamente o tempo operatório ${ }^{6}$.

A osteotomia supramaleolar é um procedimento cirúrgico comumente utilizado para corrigir deformidades congênitas ou adquiridas da região distal da tíbia ${ }^{7,8}$. Atualmente, a osteotomia supramaleolar tem sido amplamente utilizada para o tratamento da artrose assimétrica do tornozelo, como método cirúrgico preservador, visando o realinhamento e redistribuição de cargas no tornozelo e colocando-se como uma boa opção à artrodese e à artroplastia, em alguns $\operatorname{casos}^{9,10,11,12}$.

O objetivo deste relato de caso foi demonstrar o uso de um modelo de impressão tridimensional (3D) no planejamento cirúrgico de uma osteotomia supramaleolar do tornozelo em um paciente com osteoartrose tibiotársica.

\section{RELATO DE CASO}

O paciente assinou devidamente o termo de consentimento livre e esclarecido, concordando e autorizando a divulgação dos dados obtidos em prontuário, bem como as imagens e fotos exibidas neste relato de caso. Além disso, foi obtido aprovação bioética por parte de Comitê de Ética em Pesquisa (CAAE: 18913219.0.0000.5125).

Apresentamos o caso de um paciente, de 22 anos, sexo masculino, profissão vigilante. Apresentava história de trauma há dois anos, com fratura do tornozelo tratada conservadoramente em outro serviço. Relatava, no momento da consulta, dor intensa no tornozelo direito, dificuldade para deambular e praticar atividades físicas, sensação de rigidez e crepitação.

Ao exame físico, apresentava pele íntegra, sem cicatrizes, ausência de sinais de infecção, perfusão distal preservada, ausência de deficit motor e sensitivo, deformidade em valgo da região distal da perna, amplitude de movimento de $50^{\circ}$ e dor à palpação da região medial do tornozelo.

Radiografias do tornozelo direito com carga (AP e Perfil) e Tomografia Computadorizada (TC) sem carga, evidenciavam artrose pós-traumática do tornozelo direito, classificada como Takakura estágio 3A e um ângulo da superfície distal da tíbia (TAS) de $100^{09,10,13}$. (Figura 1)

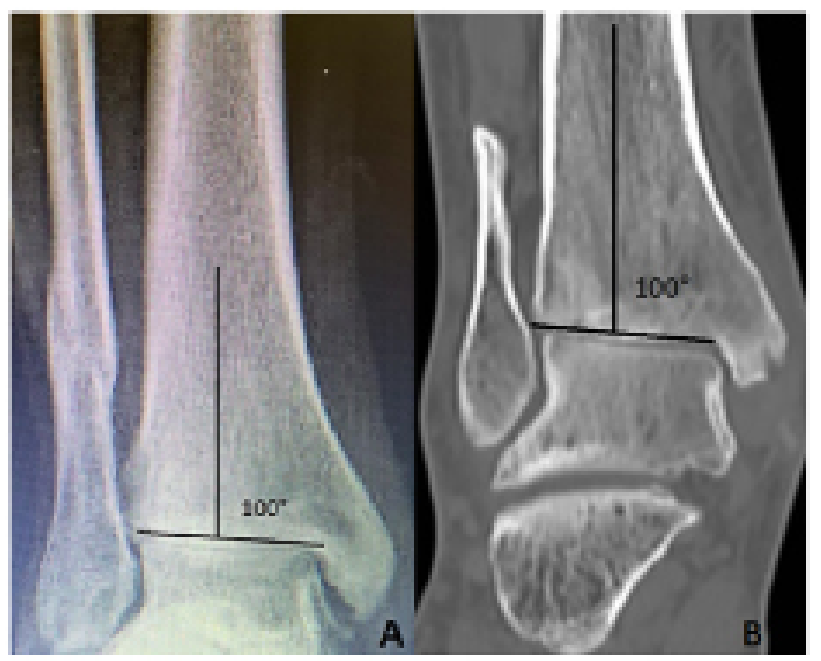

Figura 1 A) Radiografia em AP do tornozelo direito com carga. B) Corte coronal da TC sem carga pré-operatória do tornozelo direito.

O paciente relatava que havia realizado tratamento conservador no último ano de forma intensa, tendo feito mais de 100 sessões de fisioterapia, hidroginástica, infiltração de ácido hialurônico e uso de diversas medicações orais, incluindo glucosamina, diacereína e colágeno. O paciente relatava que estava descontente devido à incapacidade do tornozelo e que desejava ser submetido a um procedimento cirúrgico para melhora do quadro. 
Devido à deformidade em valgo supramaleolar e a classificação da artrose de Takakura 3A, foi optado pela realização de uma cirurgia preservadora da articulação, uma osteotomia supramaleolar (OSM) do tornozelo de cunha de subtração medial ${ }^{14}$. Essa técnica foi escolhida devido ao planejamento inicial de correção de $10^{\circ}$, o que não demanda osteotomia da fíbula para a sua complementação. Essa decisão seguiu os protocolos mais recentes para essa cirurgia, e amparou-se também nos benefícios de uma osteotomia de fechamento medial em relação a de abertura lateral, como a sua maior facilidade técnica, seus melhores índices de consolidação, a ausência de necessidade de uso de enxerto e possibilidade de planejamento acurado com modelo 3D $\mathrm{D}^{14,15,16}$.

A OSM é uma técnica cujos resultados dependem da acurácia de correção da deformidade ${ }^{16}$, e para isso, foi optado por realizar planejamento cirúrgico pré-operatório em modelo 3D. Para a confeç̧ão do modelo, a TC sem carga, cujo ângulo TAS no corte coronal foi de $100^{\circ}$, foi trabalhada utilizando-se os softwares InVesalius, Meshmixer e Slic3r. As peças foram dispostas para serem impressas, definindo-se seus parâmetros de impressão (densidade de geometria interna de $10 \%$, forma de preenchimento interno grid, altura de camada $0,25 \mathrm{~mm}$, espessura de parede 3 $\mathrm{mm}$ ). Adiante, realizou-se o fatiamento das peças, gerando o arquivo G-code. (Figura 2)

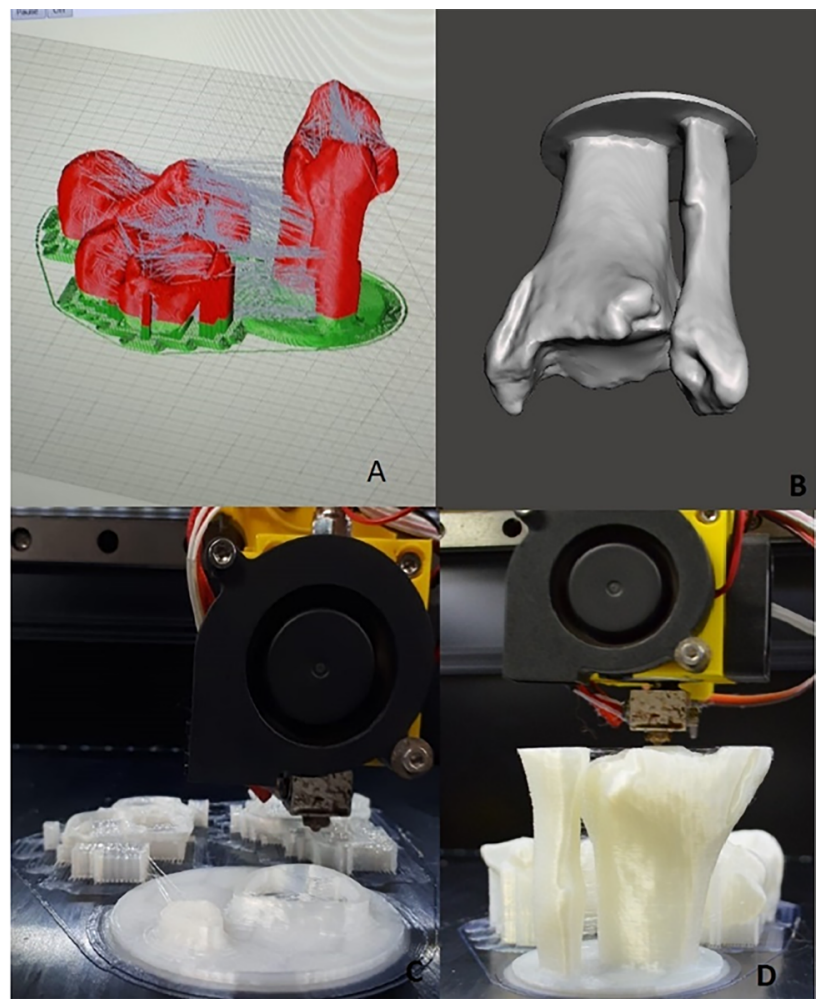

Figure 2 (A/B) Modelagem da tomografia. (C/D) Impressão da peça 3D.
Foram utilizadas as impressoras: Factor 3D P350 e Sethi 3D AIP A3. O material utilizado para impressão foi o Filamento de PLA (poliácido láctico); diâmetro da seção transversal $1,75 \mathrm{~mm}$; temperatura de extrusão $190^{\circ} \mathrm{C}$ a $210^{\circ} \mathrm{C}$. O tempo de impressão foi de 8 horas. Em laboratório, com a peça 3D, foi mensurado novamente o ângulo TAS, cujo valor foi confirmado em $100^{\circ}$.

O modelo foi então levado para o laboratório onde foi realizado o planejamento pré-operatório. Realizou-se uma osteotomia em cunha de fechamento medial da tíbia, de $1 \mathrm{~cm}$ de comprimento, $2 \mathrm{~cm}$ proximal ao ápice do maléolo medial. Estes parâmetros foram escolhidos para se atingir o CORA da deformidade e para corrigir o ângulo TAS para $90^{\circ}$, como se recomenda na literatura ${ }^{14,15,16}$. Tem-se como princípio que cada milímetro da cunha corrige um grau da deformidade ${ }^{15}$. A osteotomia foi então fixada medialmente com uma placa em T, de quatro furos, com perfil de $3,5 \mathrm{~mm}$ pré-moldada, e oito parafusos com fixação bicortical, sendo quatro parafusos corticais, tamanho $45 \mathrm{~mm}$, um parafuso cortical tamanho $30 \mathrm{~mm}$ e três parafusos bloqueados, tamanho $30 \mathrm{~mm}$. Anteriormente, foi posicionada uma placa terço tubular, de cinco furos, perfil 3,5 mm, com quatro parafusos, sendo dois bloqueados de $28 \mathrm{~mm}$, um cortical de $28 \mathrm{~mm}$ e um cortical de $40 \mathrm{~mm}$. (Figura 3). Todos os tamanhos de parafusos foram documentados e as placas moldadas.

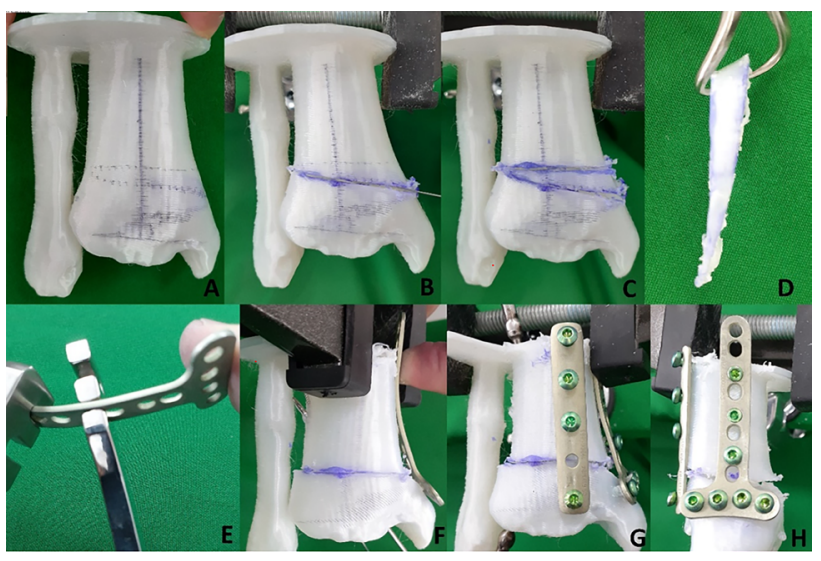

Figura 3 (A) Marcação do ângulo TAS e da cunha na peça 3D. (B/C) Corte da cunha na peça 3D. (D) Cunha. (E) Pré-moldagem da placa em T. (F) Peça 3D sem a cunha. (G) Peça 3D com os materiais de osteossíntese. $(\mathrm{H})$ Peça 3D com os materiais de osteossíntese. 
Os parafusos utilizados nesse planejamento em laboratório foram reservados para esterilização para serem utilizados in vivo. O planejamento teve duração total de 30 minutos.

Ao final do planejamento foi obtido um ângulo TAS no valor de $\left(90^{\circ}\right)^{13}$. O objetivo foi uma hipercorreção seguindo as recomendações da literatura ${ }^{14,15,16}$.

Cinco dias após o planejamento em laboratório, o paciente foi submetido à cirurgia. Sob raquianestesia, o paciente foi posicionado em decúbito dorsal. Após insuflação de torniquete, foi realizada incisão medial sobre a tíbia com extensão ao maléolo medial para realização da osteotomia supramaleolar em cunha de fechamento medial, de $1 \mathrm{~cm}$ de comprimento, 2 $\mathrm{cm}$ proximal ao ápice do maléolo medial usando os mesmos instrumentais, placa e parafusos utilizados na peça 3D. O tamanho da cunha removida foi o mesmo da obtida no planejamento (10 mm). (Figura 4). Após o controle radioscópico, foi realizado fechamento por planos convencional e posicionamento de curativo com tala gessada. A cirurgia teve duração total de 45 minutos. O ângulo TAS na radiografia AP e perfil do tornozelo obtidos na fluoroscopia, 30 e 90 dias após cirurgia foi de $90^{\circ}$. (Figura 5 )

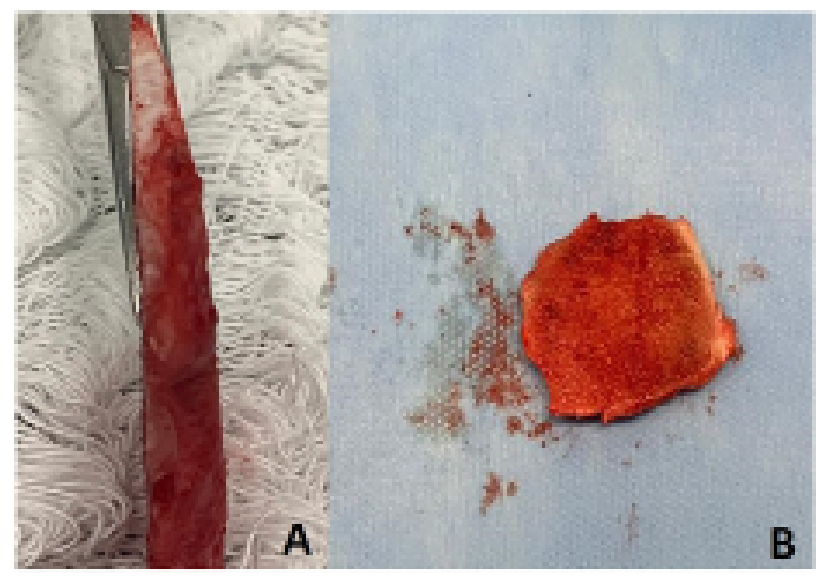

Figura 4 Desenho mostrando tamanho das placas e parafusos usados no planejamento.

Devido à estabilidade obtida com a placa medial, foi decidido no intraoperatório a não colocação da placa anterior. Utilizamos a mesma placa pré-moldada medial e os mesmos parafusos, demonstrando reprodutibilidade do planejamento cirúrgico por impressão 3D.

O pós-operatório foi conduzido em regime ambulatorial com manutenção da tala por 21 dias, retirada dos pontos no mesmo tempo e transição para

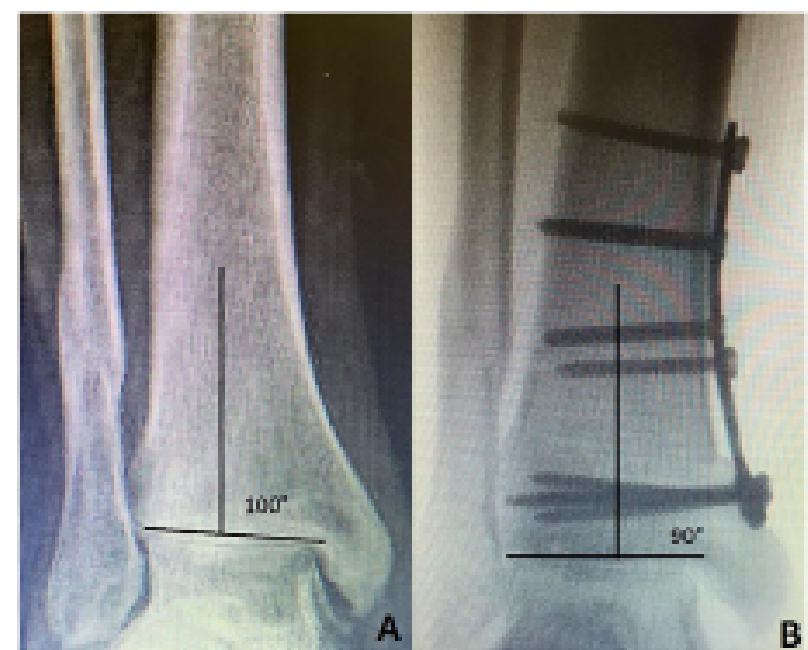

Figura 5 (A) radiografia pré-operatória em AP do tornozelo. (B) radiografia pós-operatória em $\mathrm{AP}$ do tornozelo mostrando a correção do ângulo TAS

bota tipo robofoot sem apoio. $\mathrm{O}$ apoio proprioceptivo foi iniciado com seis semanas, progredindo para apoio total com dez semanas. A fisioterapia foi iniciada na terceira semana e mantida até o final do quarto mês pós-operatório. Atividades de impacto foram liberadas após consolidação total da osteotomia e do conforto clínico do paciente, o que ocorreu aos seis meses de acompanhamento.

\section{DISCUSSÃO}

No presente relato de caso, foi obtido um ângulo TAS de $100^{\circ}$ na TC pré-operatório e na peça 3D antes do planejamento. Após o planejamento, foi obtido um valor do TAS de $90^{\circ}$, sendo este valor correspondente ao TAS na radiografia obtida no pósoperatório. Além disso, não houve necessidade de mudar número e tamanho de nenhum dos parafusos e da placa medial pré-moldada. Ao realizar uma osteotomia distal, o objetivo do cirurgião deve ser restaurar o TAS (VR: $86-92^{\circ}$ ) de volta aos valores, quando comparado com o membro contralateral e/ou ligeira hipercorreção com 2-4 $4^{\circ}$ varo ${ }^{17}$. Isto evidencia que modelos impressos em 3D são altamente precisos.

Maini et al., em estudo de caso-controle, randomizado e prospectivo, avaliaram a precisão de placas moldadas e específicas para pacientes com fratura do acetábulo. Foram incluídos 21 pacientes (dez no grupo "caso" e 11 no "controle"). No grupo "caso", modelo 3D real específico do paciente para 
o acetábulo fraturado foi confeccionado utilizando tecnologia de prototipagem rápida e pré-moldagem de placas no pré-operatório. O grupo controle foi tratado utilizando placas moldadas intra-operatoriamente. Os autores observaram que houve uma redução da perda sanguínea de $100 \mathrm{ml}$, redução do tempo cirúrgico de 12 min e melhores resultados funcionais no grupo "caso"18. Os autores concluíram que placas confeccionadas para cada paciente, utilizando modelos impressos em 3D, são superiores durante o período intraoperatório por serem mais precisas.

Bagaria et al., em um trabalho sobre o tratamento de fratura complexa do acetábulo, calcâneo e côndilo medial do fêmur (fratura de Hoffa), utilizando planejamento préoperatório semelhante ao anterior, relataram melhora no entendimento da configuração da fratura e obtiveram uma redução quase anatômica. Além disso, os autores acreditam que houve indiretamente redução do tempo cirúrgico, diminuição da necessidade de anestésico e diminuição da perda de sangue intraoperatória ${ }^{19}$. Resultados semelhantes foram encontrados também por outros autores ${ }^{20,21}$. Em nosso estudo, constatamos a precisão obtida com o planejamento tridimensional, e acreditamos também que houve diminuição do tempo cirúrgico e da perda sanguínea, porém, não foi possível quantificar esses dados.

Observa-se um aumento do uso dos modelos impressos em 3D no Brasil, não somente na medicina, mas também na medicina veterinária, odontologia e outras áreas ${ }^{22,23,24}$. Os avanços na área médica já permitem a obtenção de redes de vasos sanguíneos e de organelas hepáticas bioimpressas, utilizadas para treinamento de cirurgiões e estudos de toxicidade de drogas, respectivamente. Houve avanços também na confecção de órteses personalizadas para lesões corporais ${ }^{25,26,27}$.

Optamos por não utilizar a placa anterior no intraoperatório por não acreditarmos que ela acrescentaria estabilidade ao sistema (osso-material de osteossíntese). O tamanho dos parafusos utilizados foi determinado de forma objetiva através da medição entre as duas corticais.

Enfatizamos a necessidade de uma cuidadosa avaliação dos implantes antes do procedimento cirúrgico, visto que durante o planejamento existe o risco de danificá-los. Uma opção seria utilizar a placa modelo apenas como template, porém, o aumento nos custos pode inviabilizar o procedimento.

Destacamos aqui algumas das limitações apresentadas por este estudo. Enfatizamos a necessidade da realização de estudos, como coortes maiores e prospectivos, para que aspectos como tempo cirúrgico, acurácia de medidas, perda sanguínea e estabilidade de implantes, possam ser avaliados. Outro aspecto a ser considerado consiste na escolha do material a ser utilizado na confeç̧ão da peça 3D. O ácido polilático (PLA), material utilizado neste caso, apesar de ser biodegradável e ter um custo baixo, tem baixa resistência às forças de tensão, sendo um material frágil e pouco elástico, assim como o Polietileno Tereftalato (PET) ${ }^{28,29}$. Essa propriedade limita seu uso em situações que requeiram deformação plástica e altos níveis de estresse, como a fixação de fraturas com parafusos e placas ${ }^{30}$.

\section{CONCLUSÃO}

Peças 3D podem se demonstrar altamente precisas e contribuir para o planejamento de cirurgias ortopédicas, agregando benefícios diretos, como acurácia na correção de deformidades, modelagem da placa e escolha do tamanho dos parafusos.

\section{REFERÊNCIAS}

1. Hull, C. W. Apparatus for production of three-dimensional objects by stereolithography (1996).

2. Collin E Pehde, John Bennett, Brad Lee Peck, Logan Gull- Development of a 3-D Printing Laboratory for Foot and Ankle Applications. Clin Podiatr Med Surg. 2020 Apr;37(2):195-213. doi: 10.1016/j.cpm.2019.12.011.

3. Akhil Sharma, Kyle S Kirkland, Robert M Holloway, Selene G Parekh Incorporating 3D Printing Into Your Practice: Lessons Learned. Foot Ankle Spec. 2020 Dec 20;1938640020980912. doi: $10.1177 / 1938640020980912$.

4. Wang L, Ye X, Hao Q, Chen $Y$, Chen $X$, Wang $H$, et al. Comparison of two three-dimensional printed models of complex intracranial aneurysms for surgical simulation. World Neurosurg. 2017;103:671-679.

5. Waran V, Narayanan V, Karuppiah R, Owen SL, Aziz T. Utility of multimaterial 3D printers in creating models with pathological entities to enhance the training experience of neurosurgeons. J Neurosurg. 2014;120:489-492.

6. Feng Shuang, MD, Wei Hu, MD, Yinchu Shao, MD, Hao Li, MD, and Hongxing Zou, MD. Treatment of Intercondylar Humeral Fractures With 3D-Printed Osteosynthesis Plates. Medicine _ Volume 95, Number 3, January 2016. 
7. Abraham E, Lubicky JP, Songer MN, et al. Supramalleolar osteotomy for ankle valgus in myelomeningocele. J Pediatr Orthop 1996;16(6):774-81.

8. Nicol RO, Menelaus MB. Correction of combined tibial torsion and valgus deformity of the foot. J Bone Joint Surg $\mathrm{Br} 1983 ; 65(5): 641-5$.

9. Takakura Y, Tanaka Y, Kumai T, Tamai S. Low tibial osteotomy for osteoarthritis of the ankle. Results of a new operation in 18 patients. J Bone Joint Surg $\mathrm{Br}$ 1995;77(1):50 -4. 486 R.A. Benthien, M.S. Myerson / Foot Ankle Clin N Am 9 (2004) 475-487

10. Takakura $Y$, et al. Results of opening-wedge osteotomy for the treatment of a post-traumatic varus deformity of the ankle. J Bone Joint Surg Am 1998;80(2):213 -8.

11. Stamatis ED, Cooper PS, Myerson MS. Supramalleolar osteotomy for the treatment of distal tibial angular deformities and arthritis of the ankle joint. Foot Ankle Int $2003 ; 24(10): 754-64$

12. Tarr RR, Resnick CT, Wagner KS, et al. Changes in tibiotalar joint contact áreas following experimentally induced tibial angular deformities. Clin Orthop 1985; 199:72-80.

13. Mangone PG. Distal tibial osteototomies for the treatment of foot and ankle disorders. Foot Ankle Clin. 2001;6(3):583-97.

14. Beat Hintermann, MD, Markus Knupp, MD,Alexej Barg, MD. Supramalleolar Osteotomies for the Treatment of Ankle Arthritis. Journal of the American Academy of Orthopaedic Surgeons. July 2016, Vol 24, No 7.

15. Adam S. Becker, MDa,b,Mark S. Myerson, MDa,*. The Indications and Technique of Supramalleolar Osteotomy. Foot Ankle Clin N Am 14 (2009) 549-561 doi:10.1016/j.fcl.2009.06.002.

16. Ross A. Benthien, MD, Mark S. Myerson, MD*. Supramalleolar osteotomy for ankle deformity and arthritis R.A. Benthien, M.S. Myerson / Foot Ankle Clin N Am 9 (2004) 475-487.

17. Hintermann B, Barg A, Knupp M: Corrective supramalleolar osteotomy for malunited pronationexternal rotation fractures of the ankle. J Bone Joint Surg $\mathrm{Br} 2011 ; 93(10): 1367-1372$.

18. L. Maini1 - A. Sharma1,3 - S. Jha2 - A. Sharma1 - A. Tiwari1. Three-dimensional printing and patient-specific pre-contoured plate: future of acetabulum fracture fixation?. Eur J Trauma Emerg Surg. DOI 10.1007/ s00068-016-0738-6.

19. Bagaria V, Deshpande S, Rasalkar DD, Kuthe A, Paunipagar BK. Use of rapid prototyping and threedimensional reconstruction modeling in the management of complex fractures. Eur J Radiol. 2011;80:814-20.

20. Qipeng Wu, Tao Yu, Bo Lei, Wenjie Huang, Ruokun Huang. A New Individualized Three-Dimensional Printed Template for Lateral Ankle Ligament Reconstruction. Med Sci Monit. 2020 Mar 5;26:e922925. doi: 10.12659/ MSM. 922925.
21. Anil Murat Ozturk, Onur Suer, Istemihan Coban, Mehmet Asim Ozer, Figen Govsa. Three-Dimensional Printed Anatomical Models Help in Correcting Foot Alignment in Hallux Valgus Deformities. Indian J Orthop. $2020 \mathrm{Apr}$ 23;54(Suppl 1):199-209. doi: 10.1007/s43465-02000110-w. eCollection 2020 Sep.

22. Reis, D. de A. L. dos, Gouveia, B. L. R., Alcântara, B. M. de, Saragiotto, B. P., Baumel, Érica E. D., Ferreira, J. S., Rosa Júnior, J. C., Oliveira, F. D. de, Santos, P. R. da S., \& Assis Neto, A. C. de. (2017). Biomodelos Ósseos Produzidos por Intermédio da Impressão 3D: Uma Alternativa Metodológica no Ensino da Anatomia Veterinária. Revista De Graduação USP, 2(3), 47-53. https://doi.org/10.11606/issn.2525-376X.v2i3p47-53.

23. Ana Luísa Bettega, AcCBC-PR, Luis Fernando Spagnuolo Brunello, AcCBC-PR, Guilherme Augusto Nazar, Giovanni Yuji Enomoto De-Luca, Lucas Mansano Sarquis, AsCBCPR, Henrique de Aguiar Wiederkehr, AcCBC-PR, José Aguiomar Foggiatto, Silvania Klug Pimentel, TCBC-PR. Simulador de dreno de tórax: desenvolvimento de modelo de baixo custo para capacitação de médicos e estudantes de medicina. Rev. Col. Bras. Cir. vol.46 no. 1 Rio de Janeiro 2019. https://doi.org/10.1590/0100$6991 \mathrm{e}-20192011$.

24. J.V.P. Bertti, E.E. Silveira, A.C. Assis Neto. Reconstrução e impressão 3D do neurocrânio de cão com o uso de tomografia computadorizada como ferramenta para auxiliar no ensino da anatomia veterinária. Arq. Bras. Med. Vet. Zootec. vol.72 no.5 Belo Horizonte Sept./Oct. 2020 Epub Nov 09, 2020. https://doi. org/10.1590/1678-4162-11209.

25. Glen S. Van Arsdell, MD,a,b Nabil Hussein, MBChB,c,d and Shi-Joon Yoo, MDc,e. Three-dimensional printing in congenital cardiac surgery-Now and the future. The Journal of Thoracic and Cardiovascular Surgery 2020 ; 1-5. https://doi.org/10.1016/j.jtcvs.2019.12.131.

26. Nguyen DG, Funk J, Robbins JB, Crogan-Grundy C, Presnell SC, Singer T, et al. Bioprinted 3D primary liver tissues allow assessment of organ-level response to clinical drug induced toxicity in vitro. PLoS One. 2016;11: 1-17. 3.

27. Liacouras P1, Garnes J, Roman N, Petrich A, Grant GT. Designing and manufacturing an auricular prosthesis using computed tomography, 3-dimensional photographic imaging, and additive manufacturing: a clinical report. J Prosthet Dent. 2011;105:78-82.

28. R. AURAS, B. HARTE, S. SELKE, An overview of polylactides as packaging materials. Macromol. Biosci. v. 4, p. 835-864, 2004.

29. RASAL, R.M.; HIRT, D.E. Toughness decrease of PLA-PHBHHx blend films upon surface-confined photopolymerization. J. Biomed. Mater. Res. Part v. 88 A, p. 1079-1086, 2008.

30. RASAL, R.M.; JANORKAR, A.V.; HIRT, D.E. Poly(lactic acid) modifications, Prog. Polym. Sci. v. 35, p. 338-356, 2010. 


\section{Conflitos de interesse:}

Nenhum dos autores possuem conflitos de interesse.

Financiamento: Nenhum.

Autor correspondente:

Ricardo Fernandes Rezende

ricardomed1992@gmail.com

Editor:

Prof. Dr. Marcelo Riberto

Recebido: 25/07/2020

Aprovado: $12 / 02 / 2021$ 